\title{
Enhanced Reactivity in Surface-Confined Water of Hierarchically Structured Polymers
}

\author{
Marco Beaumont ${ }^{\star 1,2}$, Paul Jusner ${ }^{1}$, Notburga Gierlinger ${ }^{3}$, Alistair W. T. King ${ }^{4}$, Antje \\ Potthast ${ }^{1}$, Orlando J. Rojas ${ }^{2,5}$, Thomas Rosenau ${ }^{* 1,6}$
}

\begin{abstract}
Affiliations:
${ }^{1}$ Department of Chemistry, Institute of Chemistry for Renewable Resources, University of Natural Resources and Life Sciences Vienna (BOKU), Konrad-Lorenz-Straße 24, A3430 Tulln, Austria.

2 Department of Bioproducts and Biosystems, School of Chemical Engineering, Aalto University, FI-00076 Aalto, Finland.

3 Institute for Biophysics, Department of Nanobiotechnology, University of Natural Resources and Life Sciences, Muthgasse 11, Vienna, Austria.

${ }^{4}$ Materials Chemistry Division, Department of Chemistry, University of Helsinki, Al Virtasen Aukio 1, Fl-00560 Helsinki, Finland.

${ }^{5}$ Departments of Chemical and Biological Engineering, Chemistry and Wood Science, University of British Columbia, 2360 East Mall, Vancouver, BC, V6T 1 Z4 Canada.

6 Johan Gadolin Process Chemistry Centre, Åbo Akademi University, Porthansgatan 3, Åbo/Turku FI-20500, Finland.
\end{abstract}

*Correspondence: thomas.rosenau@boku.ac.at, marcobeaumont1@gmail.com

\section{Summary}

The remarkable efficiency of biological chemical reactions is the result of evolution and many of these reactions are promoted by confined water. This has inspired significant research endeavors exploiting the potential of this special water in chemistry. However, these systems are so far limited to complex artificial solids or biphasic systems and small molecules as reactants. Here, we show that the intrinsically present surface-confined water in hierarchically structured biopolymers can be used as nanomedium to promote chemical reactions. We found in the example of cellulose fibers that confined water was actively involved in the reaction mechanism and facilitated the surface acetylation of the fiber, increasing reaction kinetics, efficiency and regioselectivity. Our findings can be regarded as proof-of-principle that the hydration layer in nanoporous polymers can be exploited as medium to promote chemical reactions at their surface. This concept can likely be extended to other polymers and various reaction systems. 


\section{Introduction}

Water as the solvent of life is ubiquitous in the chemical processes of living organisms ${ }^{1}$ and all known living systems only thrive in aqueous systems. Water controls the activity of enzymes, acts as a nucleophile and mediates charge and proton transfers in biological reactions. In the presence of water, nature enables the miraculous array of complex biochemical reactions - at high efficiency and under mild conditions. These also include processes which are unfavored under aqueous conditions ${ }^{2}$, such as dehydrations and esterifications. They do nevertheless occur due to presence of optimized enzymes ${ }^{3}$; and the fact that water is spatially confined in cells and organelles ${ }^{1,4}$. Water in confinement is restricted in mobility and features anomalous behavior, if compared to the fluid in bulk, this directly affects its chemical and physical properties ${ }^{5,6}$. Exploiting the potential of this anomalous water state for chemical reactions, has led to very important scientific contributions, which take advantage of the confined water in artificial porous solids ${ }^{7-11}$ or biphasic fluid systems ${ }^{12-14}$. Current limitations are the restrictions to small molecules as reactants, the use of organic solvents and the rather complex design of these reaction systems. Expanding this concept to naturally occurring and sustainable materials, which confine water due to their intrinsic native structure, would be a major contribution to the field. This is especially true in times of climate ${ }^{15}$ and plastic crisis ${ }^{16}$. This renders the development of sustainable reactions, and materials, most timely and necessary.

Nature's best polymeric fibers are essential structural components in biological tissues: keratin ${ }^{17}$, collagen ${ }^{18}$ and fibroin ${ }^{19,20}$ as examples for protein fibers along with cellulose ${ }^{21}$ and chitin $^{22}$ as representatives from the polysaccharide realm. All these materials are based on nano-scaled assemblies, covered by surface-confined water under ambient conditions. The cellulose fiber is used herein as representative example for this class of hierarchically structured, nanoporous biopolymers. Cellulose ${ }^{23}$, from the molecular viewpoint, has the rather simple chemical structure of $\beta$-O-1,4-linked glucopyranose repeating units that give strictly regular and rigid nanofibers, i.e. elementary fibrils, as its smallest subunit (Figure 1). 


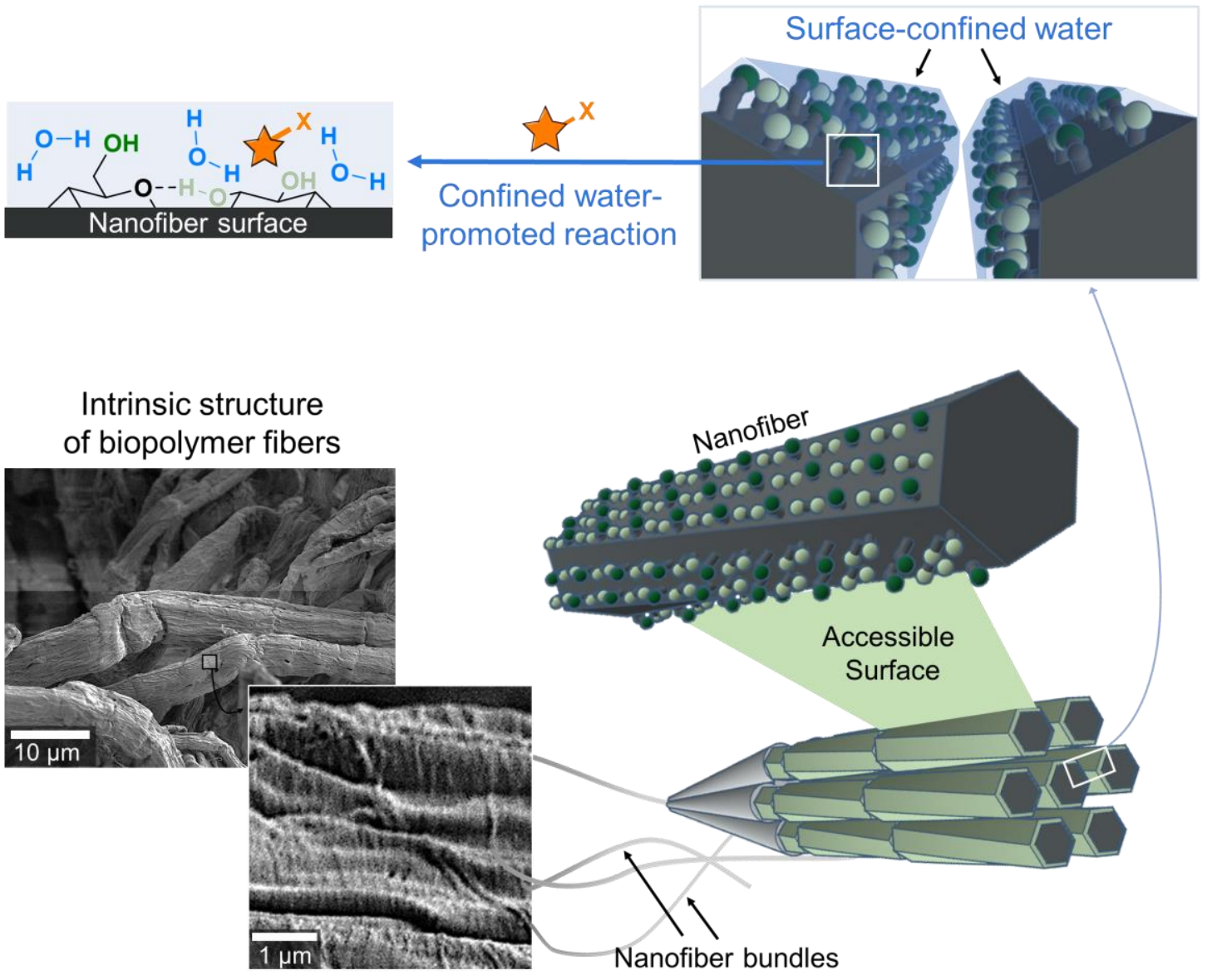

Figure 1: The hydration layer of nanoporous polymers as reaction medium. Cellulose, as example for an hierarchically structured biopolymer, is composed of individual nanofibers (i.e. elementary fibrils), organized into a micro-scaled fiber. Only the surface of these fibrils are chemically accessible (accessible $\mathrm{OH}$-groups are represented in form of light and dark green balls) and covered under ambient conditions with structurally confined water. This surface-confined water was explored as nanomedium to promote chemical reactions.

These nanofibers are composed of axially twisted crystallites ${ }^{24}$ with a chemically accessible and hydrophilic surface, which under ambient conditions is covered with a hydration layer ${ }^{25,26}$. The surface-confined water in this layer is often referred to as nonfreezing water or bound water, and can be distinguished from bulk water by its properties $^{27}$. So far, this omnipresent water has mostly been considered as a major hindrance in cellulose chemistry, when it comes to typical 'organic chemistry' reactions. The presence of water would make either reactions unfavorable or water would act as competitor to surface hydroxyl groups. As a consequence, such reactions have been mainly performed under anhydrous conditions ${ }^{28-30}$. This stays in stark contrast to recent efforts to use water as reaction promoter ${ }^{31,32}$.

With this work, we hope to induce a paradigm shift in materials chemistry by exposing the potential of confined water to promote surface reactions of nanoporous polymers. This 
principle is demonstrated here in the example of the acetylation of cellulose fibers in solid state conditions.

\section{Results}

A

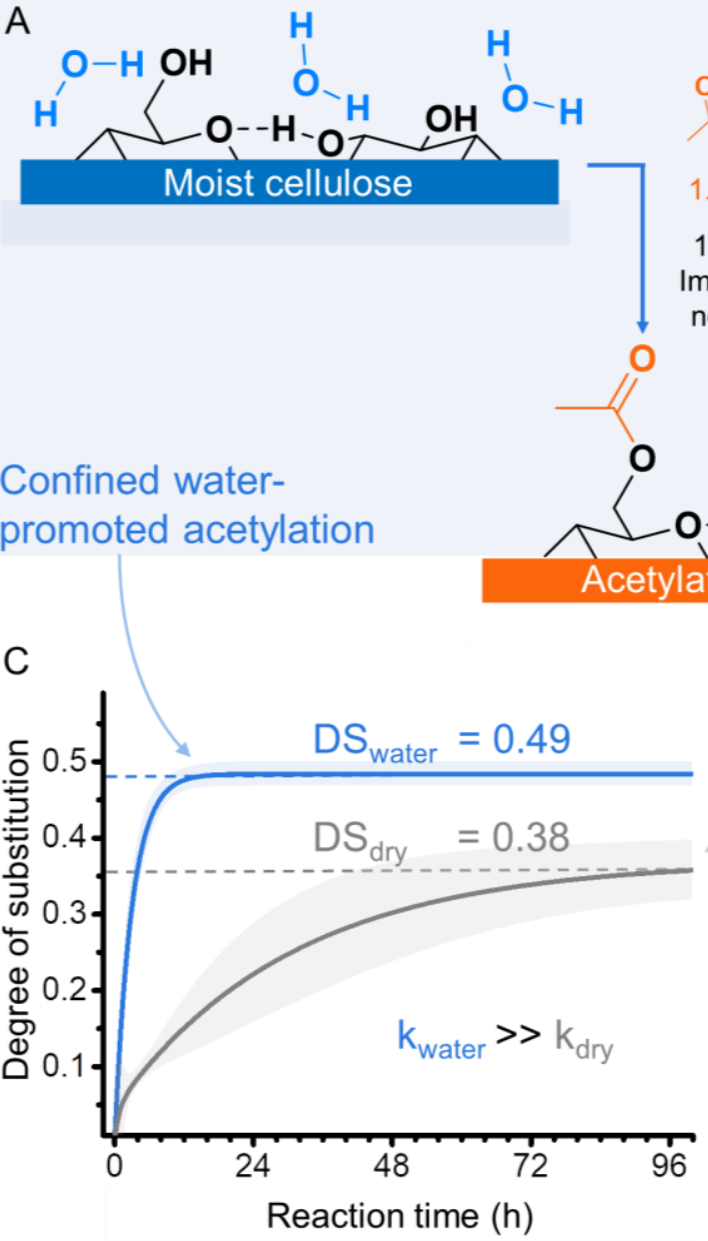

B<smiles>CC(=O)NC=N</smiles>

1.5 equiv.

$10 \mathrm{~mol} \%$ Imidazole, neat, RT

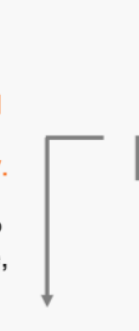<smiles>CCO</smiles>

$\mathrm{OH}$

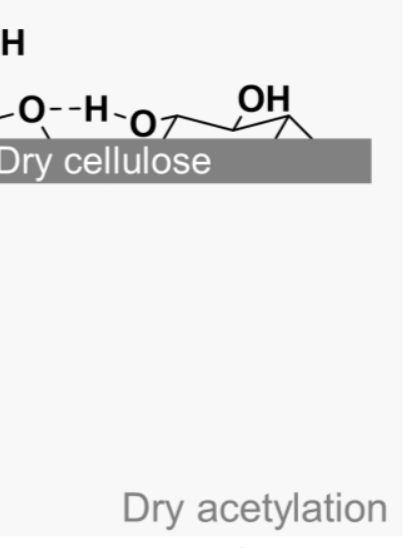

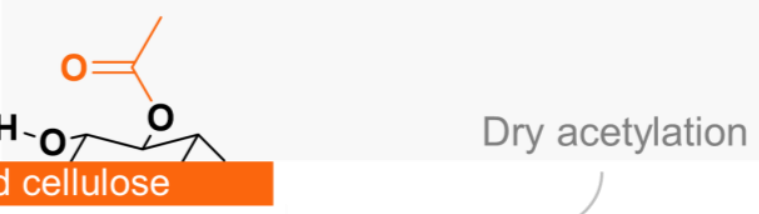

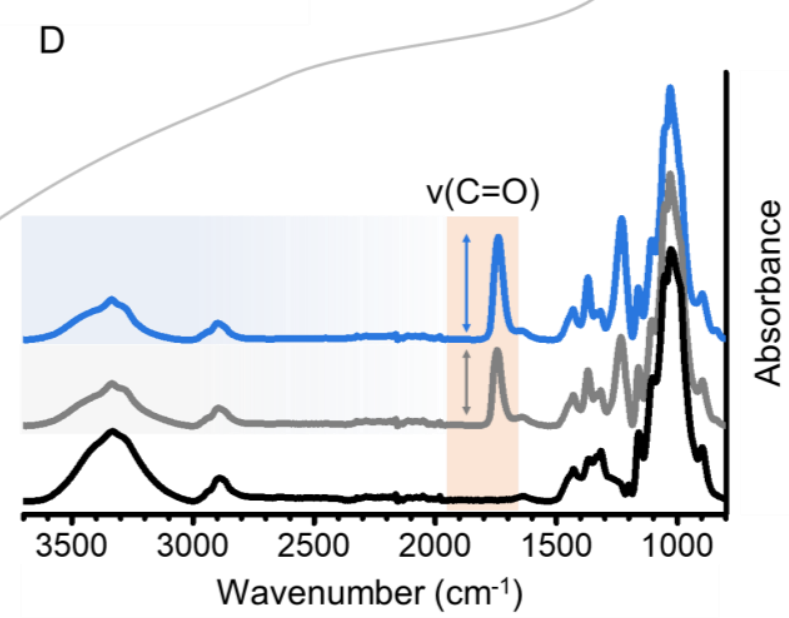

Figure 2: Acetylation in the confinement of the hydration layer. Cellulose fibers were reacted under solvent-free and heterogeneous (solid-phase) conditions with $\mathrm{N}$ acetylimidazole in presence of $7 \mathrm{wt} \%$ confined water (A, moist cellulose) and dry condition (B). The kinetics (C) and the infrared spectra (D) show that water, seemingly paradoxically, is a key player in the reaction mechanism and increases reaction rate and efficiency (95\% confidence intervals are shown in shaded color).

Wood-based cellulose fibers with a high cellulose content of $92 \%$ were used as starting material. Due to their hierarchical and porous architecture, they can structurally confine up to $0.5 \mathrm{~mL}$ of water per gram of fiber (fiber saturation point in Table S1). In case of the cellulose fiber, $c f$. Figure 1, only the surface hydroxyl groups of the elementary fibrils are accessible for chemical reactions. In general, experiments were conducted using fibers with equilibrated moisture content (EMC) of $50 \%$ relative humidity, equaling approx. 7 wt\% water (Table S1). The resulting number of water molecules is in the same stoichiometric range as the cellulose's surface hydroxyl groups and hence strongly bound to the cellulose surface. In this work, we used solely the solid reactant, $N$ - 
acetylimidazole ${ }^{33-36}$ and the catalyst imidazole to acetylate the cellulose surface. Imidazole was chosen because of its structural analogy to histidine, its associated enzyme-like catalytic behaviour ${ }^{37}$ and affinity for cellulose surfaces ${ }^{38}$.

The heterogeneous reaction of cellulose and $\mathrm{N}$-acetylimidazole was initiated by solidstate mixing using a vibratory ball mill (Figure 2A) and represents, to the best of our knowledge, the only reported true solid-state polymer acetylation method. Ball milling was chosen as it allows for mild mixing conditions, avoiding depolymerization and decrystallization of the cellulose (Figure S1). We studied the solid-state acetylation in presence of different water amounts - dry, $7 \mathrm{wt} \%, 20 \mathrm{wt} \%$ and $30 \mathrm{wt} \%$ - with respect to kinetics, reaction efficiency and regioselectivity. The calculated reaction efficiency is the ratio of the degree of substitution and the molar amount of used $\mathrm{N}$-acetylimidazole. As water is conventionally regarded as a nuisance factor in esterifications, decreasing the efficiency of this type of reaction, we expected the highest reaction efficiency in case of the dry sample. Paradoxically, we observed the contrary: as shown in Figure 2A, the presence of $7 \mathrm{wt} \%$ water in the fiber increased not only the reaction kinetics but also its efficiency. The kinetic scatter plots were exponentially fitted and resemble the shape of typical diffusion-controlled reactions ${ }^{39}$. Considering our results, the solid reactants and the solvent-free reaction conditions; we assume a reaction mechanism based on diffusion through the fiber hydration layers. The reaction is also auto-catalytic as additional formed imidazole (formed after hydrolysis or esterification of $\mathrm{N}$-acetylimidazole), can act as a base catalyst ${ }^{37}$. This has the effect of accelerating kinetics inside the fiber as the reaction proceeds (Figure S2). Increasing the water content in the cellulose sample further to higher numbers (20 wt\% and $30 \mathrm{wt} \%$ of water) than the equilibrated moisture content of $7 \mathrm{wt} \%$, did not significantly influence the kinetics of the reactions (Figure S3) but reduced the degree of substitution (DS) of the acetylated fibers and hence the reaction efficiency (Table S2). Our results, based on diffusion-edited ${ }^{1} \mathrm{H}$-NMR spectra of the acetylated fibers (Figure 3A) in the ionic liquid [P4444][OAc] ${ }^{40}$ (more details can be found in Supplemental Methods 1.8 and Figure S4), showed that that smaller amounts of secondary hydroxyl groups were modified under these conditions, i.e., increasing selectivity for the primary $6-\mathrm{OH}$. 


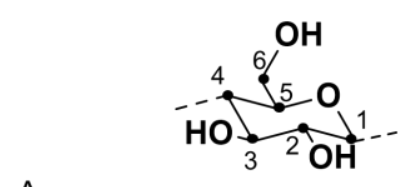

A

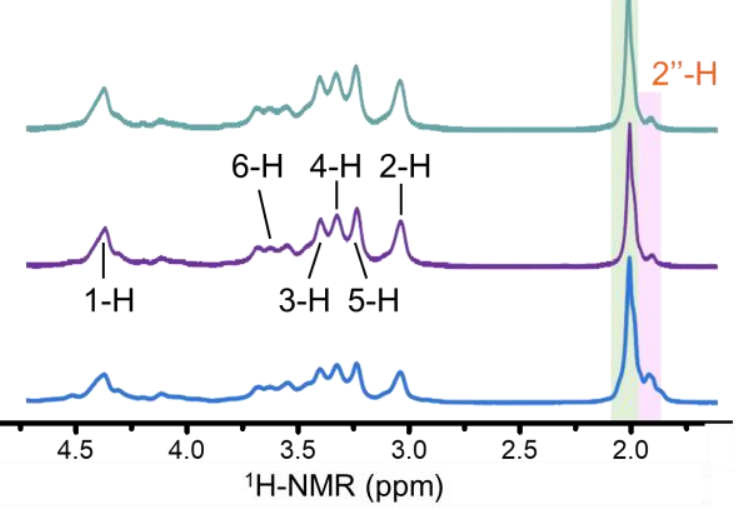

B

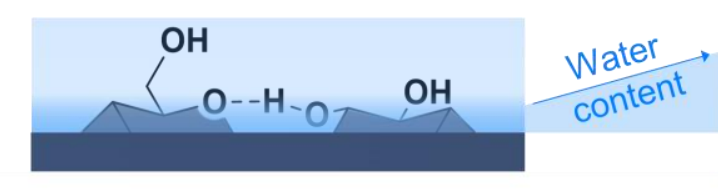

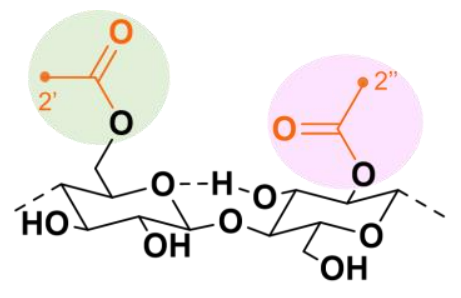

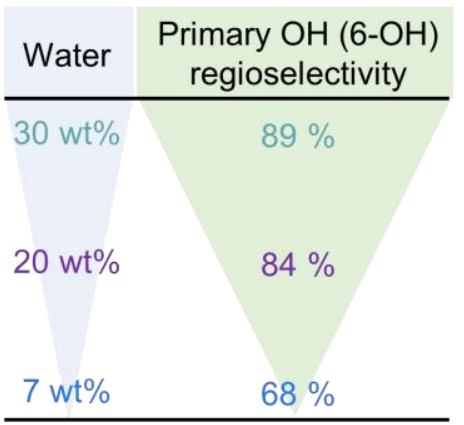

Buried" OH

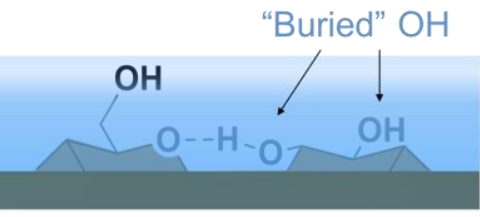

Figure 3: Water-mediated control of regioselectivity. Diffusion-edited ${ }^{1} \mathrm{H}-\mathrm{NMR}$ spectra (A) identified that increasing water allowed for more control over the acetylation regioselectivity. The primary hydroxyl group $(6-\mathrm{OH})$ was modified with up to $89 \%$ selectivity, unparalleled for a "simple" esterification. At elevated water content, the secondary hydroxyl groups are "buried" in the hydration layer rendering them chemically less accessible (B).

The regioselectivity of the reaction can thus essentially be controlled through the water content. Increasing it to $30 \mathrm{wt} \%$ elevated the selectivity towards the primary C6-hydroxyl group to $89 \%$, yielding a fiber with a DS of $0.20 \pm 0.04$. The number of modified C6groups is in the range of the estimated value ( $D S=0.25$ ) for full surface coverage, based on the 24-chain elementary fibril model and the crystallite size of the fiber (Table S1). Aligning our reasoning with previous studies of the acetylation of tyrosine in enzymes, stating that certain "buried" tyrosine residues are not chemically accessible due to hydrogen bonding.$^{41}$ We conclude that increasing the number of water molecules at the cellulose surface decreases the chemical accessibility of all hydroxyls but the secondary hydroxyls in particular, due to water-hydroxyl interactions, and/or elevates the consumption of the reactant by side-reactions with water (Figure 3B). Such selective esterification of cellulose was so far only possible in the dissolved form, i.e. under homogeneous conditions, using bulky ester groups ${ }^{42}$ or by complex multi-step syntheses, as in the case of small ester groups, such as acetyl $\left.\right|^{43}$. Therefore, apart from the confinement of the reaction system by surface water, which is a more theoretical facet of the work, this report describes the first direct method to esterify the $\mathrm{C} 6-\mathrm{OH}$ of the cellulose 
fiber surface with high selectivity under sustainable solid-state conditions. Importantly, the bulk crystallinity of cellulose was not affected but preserved (Figure S1). This is, evidently, in contrast to all methods employing homogeneous derivatization and especially the well-studied acid-catalyzed heterogeneous industrial methods for production of cellulose acetate ${ }^{28}$. Going beyond esterifications and derivatizations, only an oxidative heterogeneous method, the TEMPO-oxidation, has been able to selectively modify the superficial $\mathrm{C} 6-\mathrm{OH}^{44}$ with regioselectivities similar or only slightly superior to our approach. 

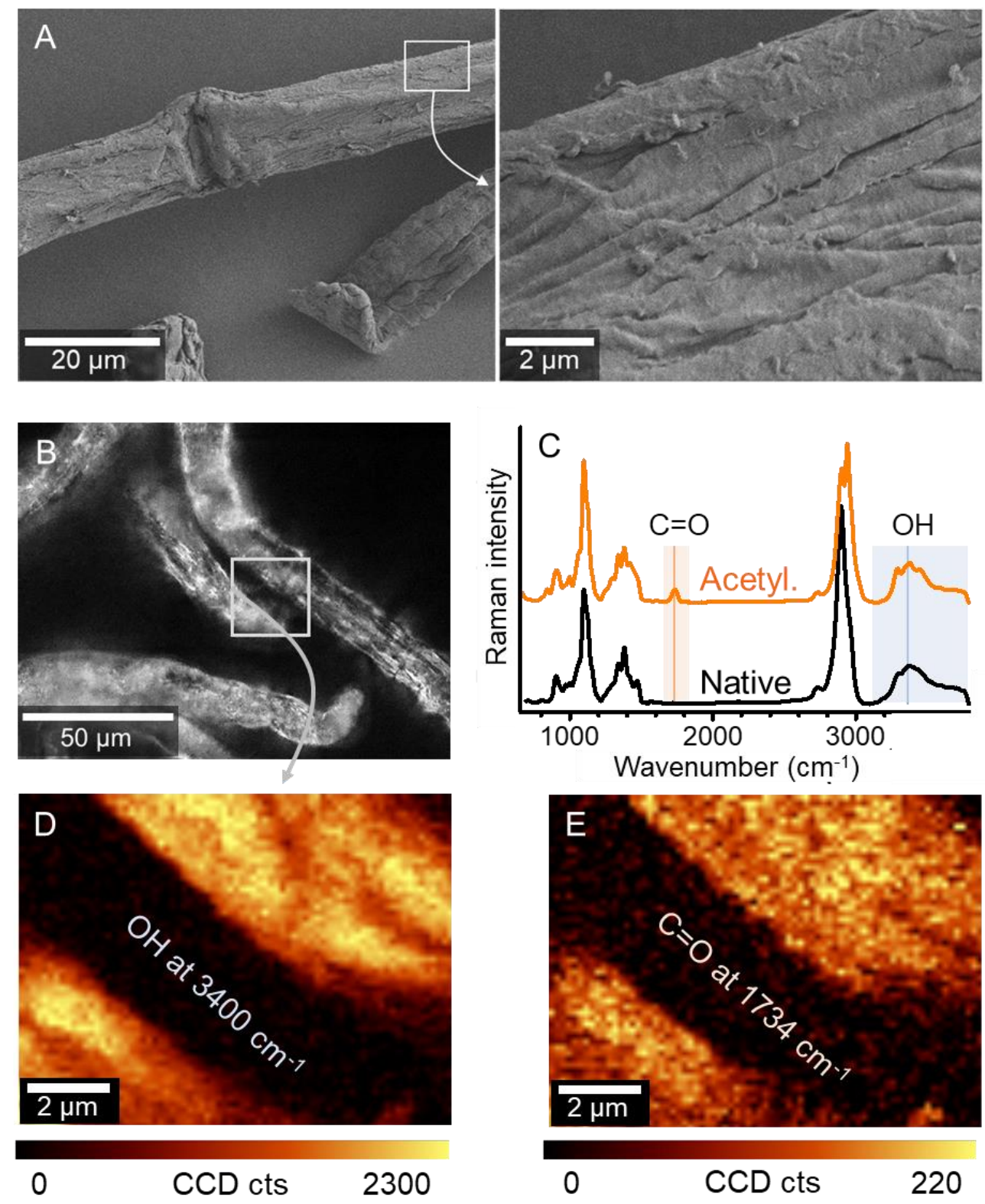

Figure 4: Effect of the confined reaction on the biopolymer surface. The confined water-promoted acetylation preserved the morphology of the native cellulose fiber, as demonstrated via scanning electron microscopy $(A$ and $B)$. The carbonyl $(C=O)$ band of the acetyl group is clearly shown in the Raman spectrum of the acetylated fiber (C). Raman mapping images of the hydroxyl $(\mathrm{OH})$ and $\mathrm{C}=\mathrm{O}$ band $(\mathrm{D})$ prove a homogeneous distribution of the acetyl groups on the cellulose fiber.

We analyzed the acetylated fibers with scanning electron, light, and Raman microscopy (Figure 4) showing that the fiber structure was not affected by the reaction and that the fiber was acetylated homogeneously, even though we conducted our acetylation in the solid state. This is another support for the involvement of the uniform surface water layer as the reaction medium. 
The preservation of the crystalline structure was further confirmed by solid-state NMR and the preservation of molecular weight by gel permeation chromatography (Figure S1).

Acetylation at EMC yielded a DS of $0.49 \pm 0.04$ corresponding to the theoretical number of accessible C6- and C2-hydroxyls of approx. $0.5 \mathrm{mmol} / \mathrm{mmol}$ (Table S1). The acetylation at C3 position seems to be negligible under our conditions and this is consistent with the literature, since the $\mathrm{C} 3-\mathrm{OH}$ is less accessible and hardly reactive due to its intra-molecular hydrogen bond with the cyclic oxygen atom of the neighboring cellulose monomer unit ${ }^{45,46}$ (see chemical structure in Figure 2A). We further studied acetylation using lower equivalents of $\mathrm{N}$-acetylimidazole ( 0.3 eq.), demonstrating a reaction efficiency of up to $79 \%$ (at EMC) (Table S2). This efficiency is remarkable for a bulk-solvent-free and heterogeneous reaction (but still containing significant surface water). It is significantly higher than the solvent-based acetylation of cellulose with $\mathrm{N}$ acetylimidazole ${ }^{36}$ and comparable to the most efficient acetylations of cellulose under homogeneous conditions ${ }^{47,48}$. While increasing the amount of $\mathrm{N}$-acetylimidazole decreases the reaction efficiency and the reaction rate (Table S2 and Figure S3), it seems that the increased reaction time and amount of $\mathrm{N}$-acetylimidazole elevates the frequency of side reactions. This includes hydrolysis of $\mathrm{N}$-acetylimidazole, as a major side reaction, which is also imidazole-catalysed ${ }^{37}$; and depletes our reaction system of its "foundation" through water consumption.
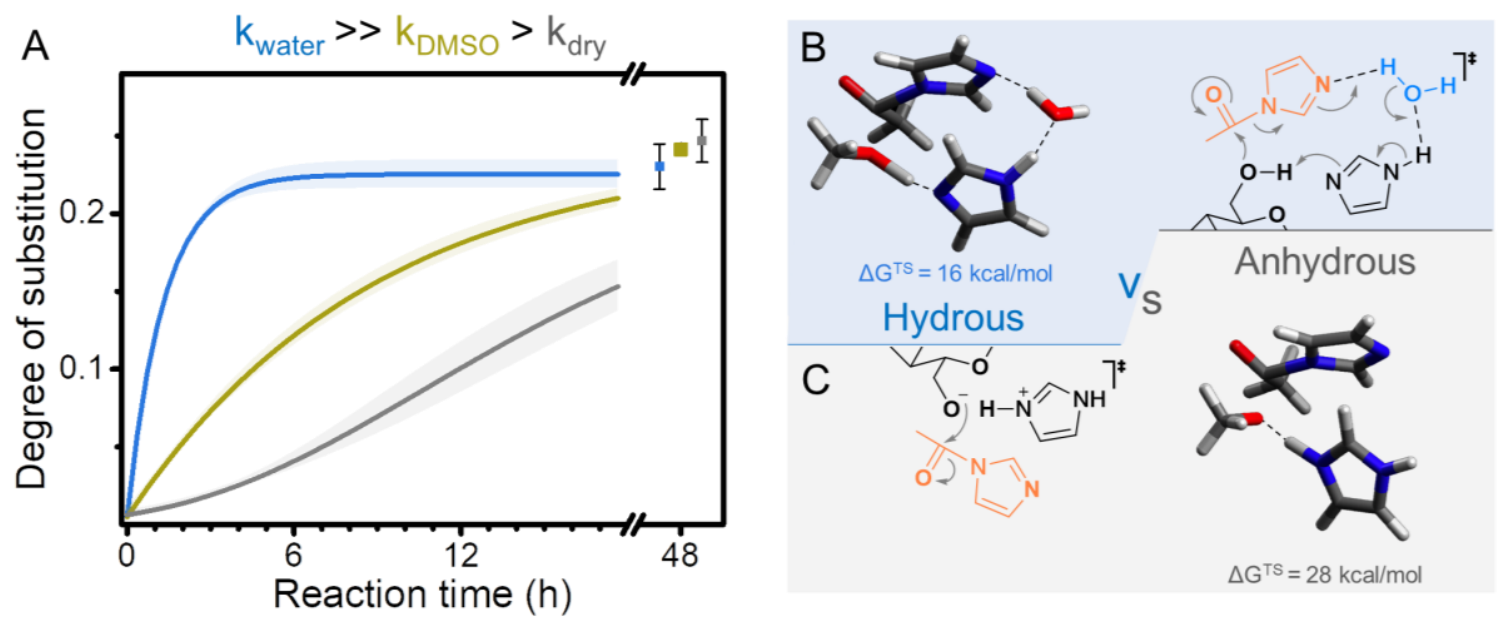

Figure 5: Mechanism of the confined water-promoted surface reaction. Comparison of acetylation kinetics of fibers with 0.3 eq. of $\mathrm{N}$-acetylimidazole under different conditions (A): Moist fibers (blue line), DMSO-wetted fibers (golden line) and dried fibers (grey line). The significantly higher reaction rate in presence of water is reasoned by an involvement of water in the mechanism of the surface reaction (B). As calculated using methanol as model primary alcohol, the presence of water significantly reduced the Gibb's free energy of activation $\left(\Delta G^{T S}\right)$ (B and $\left.C\right)$. Blue and golden lines were fitted with exponential fits and grey line was fitted with sigmoidal SGompertz function fit (R-squares > 0.99). 95\% confidence bands of the respective fits are shown in shaded color. 
We further studied the influence of water in a reaction with 0.3 equivalents of $\mathrm{N}$ acetylimidazole and obtained similar results as in the case of 1.5 equivalents: The presence of a water hydration layer (EMC) led to significantly higher reaction kinetics than the dry fiber (dry, approx. 0 wt\% moisture content) (Figure 5A). Additionally, we compared the results to an experiment with a water-free but DMSO-wetted fiber (using the same volume of DMSO as water in the fiber at EMC); this experiment was chosen to reveal if water is directly involved in the reaction mechanism or has only physical effects, such as the solvation of the reactant or a swelling effect to increase the fibers' accessibility. As shown in Figure $\mathbf{5 A}$, the presence of DMSO increased the reaction rate in comparison to the dry fiber, indicating swelling was important. However, although $\mathrm{N}$ acetylimidazole is freely soluble in $\mathrm{DMSO}^{49}$ and DMSO is a strong cellulose swelling agent ${ }^{50}$, the reaction rate in the case of the DMSO-fiber was still lower than that of the moist fiber at EMC. This indicates that the surface-confined water is not only acting as solvent medium increasing the swelling, and thus diffusion of the reactant in the fiber, but is also directly implicated in the chemical reaction mechanism. It is reported that water is involved in proton transfer reactions from imidazole groups 37,51 . We assumed that water, as protic solvent, is facilitating the proton transfer in the transition state from the cellulose acyl imidazolium intermediate (as schematically shown Figure 5B) to the imidazole base catalyst. This transition state involving a proton transfer of water in a concerted reaction mechanism was further validated through a gas-phase computational study of the acetylation of methanol (as the simplest alcohol model compound). Without water: the Gibb's free energy of activation ( $\Delta \mathrm{G}^{\mathrm{TS}}$ ) was approximated to $28 \mathrm{kcal} / \mathrm{mol}$. Addition of water relieved the strain in the transition state geometry and, therefore, reduced tremendously $\Delta \mathrm{G}^{\mathrm{TS}}$ to $16 \mathrm{kcal} / \mathrm{mol}$ (Figure 5B-C). Clearly having a small amount of water in the reaction is advantageous for esterification kinetics (due to reduced strain during proton transfer). More details of the computational results can be found in Supplemental Methods 1.9. 


\section{Discussion}

Cellulose fibers, as well as other nanoporous biopolymers, are hygroscopic and intrinsically confine water in its fibrillar structure. Our data shows, for the first time, that this confined water can be exploited as nanomedium to promote chemical reactions.

In comparison to a dry acetylation, surface-confined water elevated the reaction efficiency and increased the reaction rate of the cellulose surface acetylation with $\mathrm{N}$ acetylimidazole. In addition, the chemical accessibility of the cellulose hydroxyl groups and the regioselectivity of the reaction can be controlled by the amount of confined water on the nanofiber surface, enabling the largely regioselective surface acetylation of cellulose. Modelling of the reaction transition states revealed that the presence of water reduces the activation barrier of the acetylation, by avoiding constrained geometries and enabling a concerted proton transfer mechanism. This, evidently, is in stark contrast to the unfavorable interference of bulk water with conventional esterifications.

More broadly, we demonstrate that confining reactions inside the hydration layer of nanoporous polymers is a feasible concept to promote reactions at the polymer surface. We believe that this work can be adapted to a broad range of polymers and reactions and will lead to significant advances in the general field of materials chemistry, analytics and catalysis. 


\section{Methods}

The cellulose fibers, TCF-bleached beech sulphite dissolving pulp, were supplied by Lenzing AG (Austria). Properties and specification of the pulp are listed in Table S1. The used chemicals were, if not otherwise noted, purchased from Sigma-Aldrich (SigmaAldrich Chemie $\mathrm{GmbH}$, Munich, Germany) with a purity of at least $99 \%$. N-Acetylimidazole was supplied in a purity of $\geq 98 \%$ from $\mathrm{TCl}$ ( $\mathrm{TCl}$ Deutschland $\mathrm{GmbH}$, Germany).

Pre-treatment. Imidazole and cellulose fibers were pre-treated before the solid-state acetylation. The imidazole flakes were pulverized for 2 min in a coffee grinder (KSM 2 Grinder 4041, Braun GmbH, Germany) and stored under exclusion of moisture. The cellulose fibers $(2 \mathrm{~g})$ were pre-treated in a vibratory ball mill (Retsch CryoMill, Retsch $\mathrm{GmbH}$, Germany) for $15 \mathrm{~min}$ at $25 \mathrm{~Hz}$ in a $50 \mathrm{~mL}$ vessel using 5 grinding balls (stainless steel) with a diameter of $0.5 \mathrm{~cm}$ and one with a diameter of $1 \mathrm{~cm}$ (this pre-treatment only increased the initial reaction kinetics and is not mandatory). The fluffy fibers were finally equilibrated at a relative humidity $(\mathrm{RH})$ of $50 \%$, the equilibrated moisture content at 50 $\% \mathrm{RH}(\mathrm{EMC})$ was determined gravimetrically equaling approximately $7 \mathrm{wt} \%$. Dry cellulose fibers were obtained by vacuum drying at $40{ }^{\circ} \mathrm{C}$ for $48 \mathrm{~h}$ (dry sample). The dry sample was used to prepare fibers with different moisture contents than EMC by mixing and equilibrating the fibers in a closed vessel with the respective amount of water for at least $24 \mathrm{~h}$. The DMSO sample was prepared by equilibrating the dry sample with dry DMSO (using the same volume as the moisture in the EMC sample, i.e. $7 \% \mathrm{v} / \mathrm{w}$ ).

Solid-state acetylation with $\mathrm{N}$-acetylimidazole. Cellulose fibers at EMC $(1.0 \mathrm{~g}$ dry mass, $6.2 \mathrm{mmol}, 1 \mathrm{Eq})$ were mixed with the respective amount of $N$-acetylimidazole i.e. $0.3 \mathrm{Eq}(0.20 \mathrm{~g}, 1.85 \mathrm{mmol}), 1.0 \mathrm{Eq}(0.68 \mathrm{~g}, 6.17 \mathrm{mmol})$ or $1.5 \mathrm{Eq}(1.02 \mathrm{~g}, 9.25 \mathrm{mmol})$ in a $0.25 \mathrm{~mL}$ grinding jar. $10 \mathrm{~mol} \%$ imidazole (based on the amount of $N$-acetylimidazole) and stainless-steel grinding balls $(17.7 \mathrm{~g}, 0.5 \mathrm{~cm}$ diameter) were added and milled for 0.5 $\mathrm{h}$ at $25 \mathrm{~Hz}$ in a vibratory ball mill (Retsch CryoMill, Retsch $\mathrm{GmbH}$, Germany). After milling, the samples were equilibrated at room temperature in a closed vessel. The reaction time was defined as the sum of the milling time and equilibration times. The acetylation was quenched by addition of a saturated aqueous solution of $\mathrm{NaHCO}_{3}$. The acetylated fibers were finally washed carefully with $\mathrm{DI}$ water and dried at $105^{\circ} \mathrm{C}$ for further analysis. The acetylation was performed at various conditions as listed in Table S2 to study the influence of confined water and the amount of $\mathrm{N}$-acetylimidazole on the fiber acetylation. The DS (or degree of acetylation) was determined using infrared spectroscopy in attenuated total reflection mode (PerkinElmer Frontier IR single-range spectrometer (PerkinElmer Inc., USA)) using the calibration curve in Figure S5. Further details can be found in the Supplemental Methods. 


\section{References}

1. Ball, P. (2017). Water is an active matrix of life for cell and molecular biology. PNAS 114, 13327-13335.

2. Marsden, S.R., Mestrom, L., McMillan, D.G.G., and Hanefeld, U. (2019).

Thermodynamically and Kinetically Controlled Reactions in Biocatalysis - from Concepts to Perspectives. ChemBioChem, 426-437.

3. Küchler, A., Yoshimoto, M., Luginbühl, S., Mavelli, F., and Walde, P. (2016). Enzymatic reactions in confined environments. Nature Nanotech 11, 409-420.

4. Weckhuysen, B.M., Kitagawa, S., and Tsapatsis, M. (2018). Reactions in Confined Spaces. ChemPhysChem 19, 339-340.

5. Chakraborty, S., Kumar, H., Dasgupta, C., and Maiti, P.K. (2017). Confined Water: Structure, Dynamics, and Thermodynamics. Acc. Chem. Res. 50, 2139-2146.

6. Kumar, P., Han, S., and Stanley, H.E. (2009). Anomalies of water and hydrogen bond dynamics in hydrophobic nanoconfinement. J. Phys.: Condens. Matter 21, 504108.

7. Sipponen, M.H., Farooq, M., Koivisto, J., Pellis, A., Seitsonen, J., and Österberg, M. (2018). Spatially confined lignin nanospheres for biocatalytic ester synthesis in aqueous media. Nat Commun 9, 1-7.

8. Saha, K., Deka, J., Hens, S., Saikia, S., and Raidongia, K. (2018). Chemical reactions under the nanofluidic confinement of reconstructed lamellar membranes. J. Mater. Chem. A 6, 22931-22939.

9. Muñoz-Santiburcio, D., and Marx, D. (2017). Chemistry in nanoconfined water. Chemical Science 8, 3444.

10. Manabe, K., and Kobayashi, S. (2002). Dehydrative Esterification of Carboxylic Acids with Alcohols Catalyzed by Polymer-Supported Sulfonic Acids in Water. Advanced Synthesis \& Catalysis 344, 270-273.

11. Manabe, K., Sun, X.-M., and Kobayashi, S. (2001). Dehydration Reactions in Water. Surfactant-Type Brønsted Acid-Catalyzed Direct Esterification of Carboxylic Acids with Alcohols in an Emulsion System. J. Am. Chem. Soc. 123, 10101-10102.

12. Kreft, O., Prevot, M., Möhwald, H., and Sukhorukov, G.B. (2007). Shell-in-Shell Microcapsules: A Novel Tool for Integrated, Spatially Confined Enzymatic Reactions. Angewandte Chemie International Edition 46, 5605-5608.

13. S. Koblenz, T., Wassenaar, J., and H. Reek, J.N. (2008). Reactivity within a confined selfassembled nanospace. Chemical Society Reviews 37, 247-262.

14. Song, C.E., Park, S.J., Hwang, I.-S., Jung, M.J., Shim, S.Y., Bae, H.Y., and Jung, J.Y. (2019). Hydrophobic chirality amplification in confined water cages. Nat Commun 10,1-9. 
15. Lenton, T.M., Rockström, J., Gaffney, O., Rahmstorf, S., Richardson, K., Steffen, W., and Schellnhuber, H.J. (2019). Climate tipping points - too risky to bet against. Nature 575, 592-595.

16. The European Parliament and the council of the European Union (2019). Directive (EU) 2019/904 of the European Parliament and of the Council of 5 June 2019 on the reduction of the impact of certain plastic products on the environment.

17. Wang, B., Yang, W., McKittrick, J., and Meyers, M.A. (2016). Keratin: Structure, mechanical properties, occurrence in biological organisms, and efforts at bioinspiration. Progress in Materials Science 76, 229-318.

18. Gautieri, A., Vesentini, S., Redaelli, A., and Buehler, M.J. (2011). Hierarchical Structure and Nanomechanics of Collagen Microfibrils from the Atomistic Scale Up. Nano Lett. 11, 757766.

19. Sohn, S., Strey, H.H., and Gido, S.P. (2004). Phase Behavior and Hydration of Silk Fibroin. Biomacromolecules 5, 751-757.

20. Nguyen, A.T., Huang, Q.-L., Yang, Z., Lin, N., Xu, G., and Liu, X.Y. (2015). Crystal Networks in Silk Fibrous Materials: From Hierarchical Structure to Ultra Performance. Small 11, 1039-1054.

21. Beaumont, M., Potthast, A., and Rosenau, T. (2018). Cellulose Nanofibrils: From Hydrogels to Aerogels. In Cellulose Science and Technology, T. Rosenau, A. Potthast, and J. Hell, eds. (John Wiley \& Sons, Inc.), pp. 277-339.

22. Raabe, D., Sachs, C., and Romano, P. (2005). The crustacean exoskeleton as an example of a structurally and mechanically graded biological nanocomposite material. Acta Materialia 53, 4281-4292.

23. French, A.D., Pérez, S., Bulone, V., Rosenau, T., and Gray, D. (2018). Cellulose. In Encyclopedia of Polymer Science and Technology (American Cancer Society), pp. 1-69.

24. Majoinen, J., Hassinen, J., Haataja, J.S., Rekola, H.T., Kontturi, E., Kostiainen, M.A., Ras, R.H.A., Törmä, P., and Ikkala, O. (2016). Chiral Plasmonics Using Twisting along Cellulose Nanocrystals as a Template for Gold Nanoparticles. Advanced Materials 28, 5262-5267.

25. Chen, M., Coasne, B., Guyer, R., Derome, D., and Carmeliet, J. (2018). Role of hydrogen bonding in hysteresis observed in sorption-induced swelling of soft nanoporous polymers. Nat Commun 9, 3507.

26. Paajanen, A., Ceccherini, S., Maloney, T., and Ketoja, J.A. (2019). Chirality and bound water in the hierarchical cellulose structure. Cellulose 26, 5877-5892.

27. O’Neill, H., Pingali, S.V., Petridis, L., He, J., Mamontov, E., Hong, L., Urban, V., Evans, B., Langan, P., Smith, J.C., et al. (2017). Dynamics of water bound to crystalline cellulose. Scientific Reports 7, 11840.

28. Steinmeier, H. (2004). 3. Acetate manufacturing, process and technology- 3.1 Chemistry of cellulose acetylation. Macromolecular Symposia 208, 49-60. 
29. Liebert, T.F., and Heinze, T. (2005). Tailored Cellulose Esters: Synthesis and Structure Determination. Biomacromolecules 6, 333-340.

30. Habibi, Y. (2014). Key advances in the chemical modification of nanocelluloses. Chem. Soc. Rev. 43, 1519-1542.

31. Butler, R.N., and Coyne, A.G. (2010). Water: Nature's Reaction Enforcer-Comparative Effects for Organic Synthesis "In-Water" and "On-Water." Chem. Rev. 110, 6302-6337.

32. Li, G., Wang, B., and Resasco, D.E. (2020). Water-Mediated Heterogeneously Catalyzed Reactions. ACS Catal. 10, 1294-1309.

33. Riordan, J.F., Wacker, W.E.C., and Vallee, B.L. (1965). N-Acetylimidazole: A Reagent for Determination of "Free" Tyrosyl Residues of Proteins*. Biochemistry 4, 1758-1765.

34. Hagiwara, H., Morohashi, K., Suzuki, T., Ando, M., Yamamoto, I., and Kato, M. (1998). Solid State Acetylation with Acetylimidazole: Selective Protection of Primary Alcohols and Phenols. Synthetic Communications 28, 2001-2006.

35. Lu, Y., Wei, P., Pei, Y., Xu, H., Xin, X., and Pei, Z. (2014). Regioselective acetylation of carbohydrates and diols catalyzed by tetramethyl-ammonium hydroxide in water. Green Chem. 16, 4510-4514.

36. Beaumont, M., Winklehner, S., Veigel, S., Mundigler, N., Gindl-Altmutter, W., Potthast, A., and Rosenau, T. (2020). Wet esterification of never-dried cellulose: a simple process to surface-acetylated cellulose nanofibers. Green Chem., 10.1039.D0GC02116D.

37. Nothling, M.D., Ganesan, A., Condic-Jurkic, K., Pressly, E., Davalos, A., Gotrik, M.R., Xiao, Z., Khoshdel, E., Hawker, C.J., O’Mara, M.L., et al. (2017). Simple Design of an EnzymeInspired Supported Catalyst Based on a Catalytic Triad. Chem 2, 732-745.

38. Zhao, L., Smolarkiewicz, I., Limbach, H.-H., Breitzke, H., Pogorzelec-Glaser, K., Pankiewicz, R., Tritt-Goc, J., Gutmann, T., and Buntkowsky, G. (2016). Imidazole-Doped Cellulose as Membrane for Fuel Cells: Structural and Dynamic Insights from Solid-State NMR. J. Phys. Chem. C 120, 19574-19585.

39. Khawam, A., and Flanagan, D.R. (2006). Solid-State Kinetic Models: Basics and Mathematical Fundamentals. J. Phys. Chem. B 110, 17315-17328.

40. King, A.W.T., Mäkelä, V., Kedzior, S.A., Laaksonen, T., Partl, G.J., Heikkinen, S., Koskela, H., Heikkinen, H.A., Holding, A.J., Cranston, E.D., et al. (2018). Liquid-State NMR Analysis of Nanocelluloses. Biomacromolecules 19, 2708-2720.

41. Riordan, J.F., Wacker, W.E.C., and Valler, B.L. (1965). 'Buried' Tyrosyl Residues and the Activity of Trypsin. Nature 208, 1209.

42. Xu, D., Li, B., Tate, C., and Edgar, K.J. (2011). Studies on regioselective acylation of cellulose with bulky acid chlorides. Cellulose 18, 405-419.

43. Fox, S.C., Li, B., Xu, D., and Edgar, K.J. (2011). Regioselective Esterification and Etherification of Cellulose: A Review. Biomacromolecules 12, 1956-1972. 
44. Okita, Y., Saito, T., and Isogai, A. (2010). Entire Surface Oxidation of Various Cellulose Microfibrils by TEMPO-Mediated Oxidation. Biomacromolecules 11, 1696-1700.

45. Rosenau, T., Potthast, A., Hofinger, A., Bacher, M., Yoneda, Y., Mereiter, K., Nakatsubo, F., Jäger, C., French, A.D., and Kajiwara, K. (2018). Toward a Better Understanding of Cellulose Swelling, Dissolution, and Regeneration on the Molecular Level. In Cellulose Science and Technology (John Wiley \& Sons, Ltd), pp. 99-125.

46. Lindh, E.L., Bergenstråhle-Wohlert, M., Terenzi, C., Salmén, L., and Furó, I. (2016). Nonexchanging hydroxyl groups on the surface of cellulose fibrils: The role of interaction with water. Carbohydrate Research 434, 136-142.

47. Asaadi, S., Kakko, T., King, A.W.T., Kilpeläinen, I., Hummel, M., and Sixta, H. (2018). HighPerformance Acetylated loncell-F Fibers with Low Degree of Substitution. ACS Sustain Chem Eng 6, 9418-9426.

48. Wu, J., Zhang, J., Zhang, H., He, J., Ren, Q., and Guo, M. (2004). Homogeneous Acetylation of Cellulose in a New Ionic Liquid. Biomacromolecules 5, 266-268.

49. Habibian, M., Velema, W.A., Kietrys, A.M., Onishi, Y., and Kool, E.T. (2019). Polyacetate and Polycarbonate RNA: Acylating Reagents and Properties. Org Lett 21, 5413-5416.

50. Klemm, D., Philipp, B., Heinze, T., Heinze, U., Wagenknecht, W., and John Wiley \& Sons (1998). Comprehensive cellulose chemistry. Vol. 1 - Fundamentals and Analytical Methods (Wiley-VCH).

51. Shimahara, H., Yoshida, T., Shibata, Y., Shimizu, M., Kyogoku, Y., Sakiyama, F., Nakazawa, T., Tate, S., Ohki, S., Kato, T., et al. (2007). Tautomerism of Histidine 64 Associated with Proton Transfer in Catalysis of Carbonic Anhydrase. J. Biol. Chem. 282, 9646-9656.

Acknowledgements: We wish to acknowledge CSC - IT Center for Science, Finland, and Finnish Grid and Cloud Infrastructure (persistent identifier urn:nbn:fi:research-infras2016072533) for computational resources. We thank Markus Bacher, Dr. Sonja Schiehser and Dr. Caio G. Otoni for their support with NMR, GPC and DVS measurements, respectively. Dr. Blaise L. Tardy is acknowledged for conceptual insights and SEM measurement. Funding: The authors thank the Academy of Finland (Project \# 311255, 'WTF-Click-Nano') and H2020-ERC-2017-Advanced Grant "BioELCell" (788489) for funding. Author contributions: M.B. and T.R. conceived the idea and supervized the project. P.J. and M.B. contributed to general material preparation and characterization. N.G. contributed to structural characterization with Raman and light microscopy. A.W.T.K. performed NMR analysis and computational calculations. A.W.T.K., A.P., O.J.R, T.R. and M.B. provided important conceptual insights. All authors contributed to the interpretation of the results. M.B., A.W.T.K. and P.J. wrote the paper in consultation with and input of all authors.

\section{Competing interests}

Authors declare no competing interests.

\section{Data and materials availability:}


All data is available in the main text or the supplemental materials.

\section{Supplemental Materials:}

Figures S1 to S5, Tables S1 to S4, Supplemental Methods, Supplemental Data Supplemental References, Schemes S1 to S6 